\title{
Observability singularity of batch bioreactors: a solution based on high order sliding mode differentiator approach.
}

\author{
I. Haidar, J-P Barbot, A. Rapaport and M. Ghanes *
}

\begin{abstract}
This paper deals with the observability singularity problem of batch bioreactors on the positive Orthant. This singularity is overcame by the dedicated approach based on the well-known high order sliding mode differentiator proposed by Arie Levant and the resolution of simple second order equation. Nevertheless, it is difficult to distinguish between both solutions, but the second differentiation of the output gives an appropriate test procedure for choice between both solutions. Some simulation results highlight the well-founded of the proposed method.
\end{abstract}

keywords: Nonlinear observer, Observability singularity, High order sliding mode, batch bioreactor.

\section{Introduction}

An important class of bioprocesses is the batch bioreactor [7]. This type of bioprocess is mainly used in food and pharmaceutical industry [2]. It permits the cultivation of a microbial biomass on a substrate in a controlled ( $\mathrm{Ph}$, temperature) medium. The batch culture is characterized by the fact that after the initial charge of the substrate in the bioreactor and biomass innoculation, there is no inflow or outflow of the medium. The typical model characterizing the substrate biodegradation, in this case, is given by the following equations

$$
\begin{aligned}
\dot{b} & =\mu(s) b \\
\dot{s} & =-\mu(s) b
\end{aligned}
$$

where $s$ is the substrate concentration, $b$ is the biomass concentration and $\mu(\cdot)$ is the microbial growth rate function. We have assumed here, without any loss of generality, that the units in which the concentrations $s$ and $b$ are considered have been chosen such that the

*I. Haidar and J-P Barbot are with QUARTZ Laboratory EA 7393, ENSEA, 6 Avenue du Ponceau, 95014 Cergy-Pontoise, France, A. Rapaport is with MISTEA, Inra, Montpellier SupAgro, Univ Montpellier, 2 pl. Viala 34060 Montpellier, France and M. Ghanes is with LS2N (Laboratoire des Sciences du Numrique de Nantes) - UMR 6004, 1, rue de la No BP 92101, 44321 NANTES Cedex 3. yield coefficient is equal to 1 .Several models of microbial growth have been discussed in the literature. The Haldane's model is a popular one that describes the dynamics of the growth of a biomass which is inhibited by high substrate concentration [1]. This is given by the following

$$
\mu(s)=\frac{\bar{\mu} s}{K_{s}+s+\frac{s^{2}}{K_{i}}}
$$

where $\bar{\mu}, K_{s}$ and $K_{i}$ are positive parameters.

Several works in the literature aim to reconstruct the substrate concentration from (1) when only the biomass $b$ is measured (see, e. g., $[5,9,10])$. Let us underline that in $[5,9]$, a continuous-culture bioreactor was considered instead of a batch one. This observability problem becomes more difficult when dealing with nonmonotonic microbial growth function (of type (2) for example) (see, e.g., [10]). The difficulty derives from the fact that system (1) becomes unobservable. In fact, due to the non-monotonicity of $\mu$ the rank condition for local observability [6] is not everywhere satisfied.

Here, we overcame the observability problem of system (1)-(2). Using a second order sliding mode differentiator [8], we construct a convergent observer allowing to compute (in finite time) $\dot{y}$ and $\ddot{y}$, the first and second derivative of the output

$$
y=\log (b) \text {. }
$$

Knowing that it is a finite time convergence, the comparison of $\dot{y}$ and $\ddot{y}$ with the original system permits to build a test procedure allowing the exact construction of the substrate concentration by considering the original parameters system known. Note that this result is still true for a general class of Lipschitz continuous nonmonotonic positive function $\mu$ presenting an increasing decreasing branches.

\section{Problem Statement}

As system (1) is a positive system then $b$ is positive for all $t \geq 0$ as soon as $b(0)$ is positive. Thus, $\log (b)$ is considered as the output and the following change of coordinate is used $(y, s) \rightarrow(\log (b), s)$. By consequence, 
system (1) becomes

$$
\begin{aligned}
\dot{y} & =\mu(s) \\
\dot{s} & =-\mu(s) e^{y} .
\end{aligned}
$$

The observability matrix at the order 2 of system (3), given by [6]

$$
d O_{2}=\left(\begin{array}{cc}
1 & 0 \\
0 & \frac{\partial \mu(s)}{\partial s}
\end{array}\right)
$$

with

$$
\frac{\partial \mu(s)}{\partial s}=\frac{\bar{\mu}}{K_{i}} \frac{K_{s} K_{i}-s^{2}}{\left(K_{s}+s+\frac{s^{2}}{K_{i}}\right)^{2}}
$$

shows that the system has a observability singularity set (see [4])

$$
S O_{2}=\left\{(b, s) \backslash s=\sqrt{K_{i} K_{s}}\right\} .
$$

Note that the solution $s=-\sqrt{K_{i} K_{s}}$ is not a possible solution with respect to a biological purpose. In order to overcome this singularity problem, one can increase the dimension of system (3) by considering the second derivative of the output. The observability matrix in this case is given by

$$
d O_{3}=\left(\begin{array}{cc}
1 & 0 \\
0 & \frac{\partial \mu(s)}{\partial s} \\
\frac{\partial \ddot{y}}{\partial y} & \frac{\partial \ddot{y}}{\partial s}
\end{array}\right)
$$

with

$$
\frac{\partial \ddot{y}}{\partial s}=-e^{y}\left[\left(\frac{\partial \mu}{\partial s}\right)^{2}+\frac{\partial^{2} \mu}{\partial s^{2}} \mu(s)\right]
$$

and

$$
\frac{\partial^{2} \mu(.)}{\partial s^{2}}=\frac{-2 \bar{\mu}\left(K_{s} K_{i}+4 K_{s} s+s^{2}\right)}{K_{i}\left(K_{s}+s+\frac{s^{2}}{K_{i}}\right)^{3}} .
$$

Then, when $s=\sqrt{K_{s} K_{i}}$ (i.e. $\left.\frac{\partial \dot{y}}{\partial s}=0\right), \frac{\partial \ddot{y}}{\partial s}$ can be also equal to zero only if $b=0$ (i.e. $y=\log (b)=-\infty$ ). This solution is impossible in finite time with respect to the model (1). Consequently, we conclude that considering $d \mathrm{O}_{3}$, the set of singularity is:

$$
\mathrm{SO}_{3}=\emptyset \text {. }
$$

From the previous arguments, we conclude that the system is locally weakly observable every where in $\mathbb{R}^{+} \backslash\{0\} \times \mathbb{R}^{+}$.

Recall that our problem is to estimate $s$ from the measurement of $y$ (or equivalently $b$, remembering that $y=\log (b))$ and this for all $(b, s) \in \mathbb{R}^{+} \backslash\{0\} \times \mathbb{R}^{+}$. For this, we will use an observer. But as some information came from the second derivative of $y$ we will use an observer of third dimension.

\section{High order sliding mode observer}

The following high order sliding mode differentiator is considered in order to compute $y, \dot{y}, \ddot{y}$ (see [8])

$$
\begin{aligned}
& \dot{z}_{1}=z_{2}-K_{1} L^{\frac{1}{3}}\left|z_{1}-y\right|^{\frac{2}{3}} \operatorname{sign}\left(z_{1}-y\right) \\
& \dot{z}_{2}=z_{3}-K_{2} L^{\frac{1}{2}}\left|z_{1}-y\right|^{\frac{1}{3}} \operatorname{sign}\left(z_{1}-y\right) \\
& \dot{z}_{3}=-K_{3} \operatorname{Lign}\left(z_{1}-y\right)
\end{aligned}
$$

which gives the following dynamic of the observation error

$$
\begin{aligned}
& \dot{e}_{1}=e_{2}-K_{1} L^{\frac{1}{3}}\left|e_{1}\right|^{\frac{2}{3}} \operatorname{sign}\left(e_{1}\right) \\
& \dot{e}_{2}=e_{3}-K_{2} L^{\frac{1}{2}}\left|e_{1}\right|^{\frac{1}{3}} \operatorname{sign}\left(e_{1}\right) \\
& \dot{e}_{3}=-K_{3} L \operatorname{sign}\left(e_{1}\right)
\end{aligned}
$$

Where $e_{1}=z_{1}-y, e_{2}=z_{2}-\dot{y}$ and $e_{3}=z_{3}-\ddot{y}$, then the vector $\left(z_{1}, z_{2}, z_{3}\right)^{T}$ converges in finite time to $(y, \dot{y}, \ddot{y})^{T}$ because the solutions of the original system are bounded. The solution and the convergence proof of the discontinuous system (11) is in a sense of Filippov [3].

\section{Reconstruction of the substrate}

Considering that the finite time observer (10) has converged (and more particularly that $z_{2}=\dot{y}$ ), equation (2) gives the following quadratic equation:

$$
z_{2} s^{2}+\left(z_{2} K_{i}-\bar{\mu} K_{i}\right) s+z_{2} K_{s} K_{i}=0
$$

which has two real solutions

$$
\left\{\begin{array}{l}
S_{1}=\frac{-\left(z_{2} K_{i}-\bar{\mu} K_{i}\right)+\sqrt{\left(z_{2} K_{i}-\bar{\mu} K_{i}\right)^{2}-4 z_{2}^{2} K_{s} K_{i}}}{2 z_{2}}, \\
S_{2}=\frac{-\left(z_{2} K_{i}-\bar{\mu} K_{i}\right)-\sqrt{\left(z_{2} K_{i}-\bar{\mu} K_{i}\right)^{2}-4 z_{2}^{2} K_{s} K_{i}}}{2 z_{2}} .
\end{array}\right.
$$

The singularity in $\dot{y}=0$ for $S_{1}$ and $S_{2}$ is not a real singularity because this correspond to $\mu(s)=0$ and then $s=0$, so in this case $s$ is known.

Now in order to determine between $S_{1}$ and $S_{2}$ which is the solution of (3), it is enough to use the information given by $\ddot{y}$. For that, using the fact that after a finite time $\ddot{y}=z_{3}$, it is enough to determine if it is $S_{1}$ or $S_{2}$ which verifies the following equality:

$$
\ddot{y}=z_{3}=-\frac{\partial \mu}{\partial s} \mu e^{y} .
$$

From (2) and (5) we obtain:

$$
\ddot{y}=z_{3}=-\bar{\mu}^{2}\left(K_{s}-\frac{s^{2}}{K_{i}}\right) \frac{e^{y} s}{\left(K_{s}+s+\frac{s^{2}}{K_{i}}\right)^{3}} .
$$




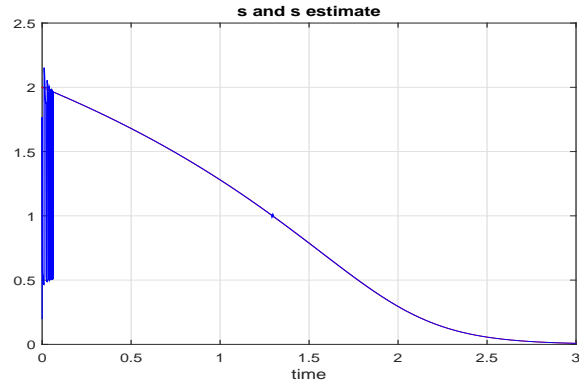

Figure 1. The substrat $s$ in red, and its estimate in blue.

This gives the following test procedure, defining the function Test

$$
\text { Test }=z_{3}+\bar{\mu}^{2}\left(K_{s}-\frac{s^{2}}{K_{i}}\right) \frac{s e^{y}}{\left(K_{s}+s+\frac{s^{2}}{K_{i}}\right)^{3}}
$$

and we chose the solution $S_{1}$ if for $s=S_{1}$ the function Test $=0$ (or if it is the closest to zero) and we chose the solution $S_{2}$ if for $s=S_{2}$ the function Test $=0$ (or if it is the closest to zero).

Remark 1 It is convenient to verify that our observer recover the singularity $\bar{s}=\sqrt{K_{S} K_{i}}$. This consist to verify that when $s=\bar{s}$, we have well $S_{1}=S_{2}=\bar{s}$. A straightforward computation leads to this end.

\section{Simulation results}

The simulation was performed on Matlab, the computation step was $h=10^{-7} h$ and a simple Euler scheme was used. The initial conditions for the system are $s(0)=2, y(0)=0.7$ and the initial condition for the differentiator are $z_{1}(0)=y(0), z_{2}(0)=0.3, z_{3}(0)=0$. The parameters for the system are $\bar{\mu}=K_{i}=K_{s}=1$ and for the differentiator are $L=90, K_{1}=6, K_{2}=12, K_{3}=8$. Figure 1 shows that the substrate was well estimated after less than 0.1 (h). Moreover, the figure 1 shows that the observability singularity point (i.e. $s=\sqrt{K_{i} K_{S}}$ ) was passed without difficulty because of the finite time convergence of the derivatives. This was emphasized in figure 2 where at this particular point which correspond to double solutions of equation (12), the test function 'hesitates' between two very closes solutions, which is only due to precision error and not the convergence of the derivatives.

\section{Conclusion}

This paper has highlighted, first the efficiency of the High Order Sliding mode observer and second the possibility to overcome an observability singularity of

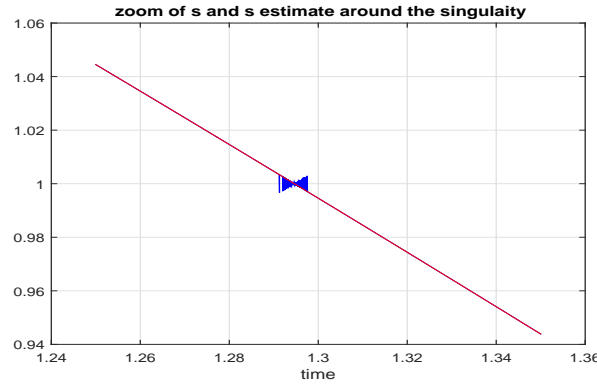

Figure 2. Zoom around the singularity of the substrat $s$ in red, and its estimate in blue.

batch bioreactors by the mean of a simple algebraic test thanks to the finite time convergence of the derivatives of the measurement signal. Our future works will be devoted to taking into account the fact that the biomass measurement is sampled. The confrontation of our observer with experimental data of $y, \dot{y}$ and $\ddot{y}$ is one of the main ends of future works.

\section{References}

[1] J. Andrews, "A mathematical model for the continuous culture of microorganisms utilizing inhibitory substrate," Biotach. Bioeng., vol. 10, pp. 707-723, 1968.

[2] J. Baranyi and T. Roberts, "Mathematics of predictive food microbiology," Int. J. Food Microbiol, vol. 26, pp. 199-218, 1995.

[3] A. F. Fillipov, Differential equations with discontinuous right-hand side. Kluwer Academic Publishers, collection : Mathematics and its Applications, 1988.

[4] M. Frunzete, J.-P. Barbot, and C. Letellier, "Influence of the singular manifold of observable states in reconstructing chaotic attractors," Physical Rev. E, vol. 86, 2012.

[5] J.-P. Gauthier, H. Hammouri, and S. Othman, "A simple observer for nonlinear systems with applications to bioreactors," IEEE Transactions on Automatic Control, vol. 37, no. 6, pp. 875-880, 1992.

[6] R. Hermann and A. Krener, "Nonlinear controllability and observability," IEEE Transactions on Automatic Control, vol. 22, no. 5, pp. 728-740, 1977.

[7] J.Pirt, Principles of microbe and cell cultivation. John Wiley and Sons, 1976.

[8] A. Levant, "Higher-order sliding modes, differentiation and output-feedback control," International Journal on automatic, vol. 76, no. 9/10, pp. 924-941, 2003.

[9] J. A. Moreno and D. Dochain, "Finite time converging input observers for nonlinear second-order systems," 52nd IEEE Conference on Decision and Control December 10-13, 2013. Florence, Italy.

[10] A. Rapaport and M. Maloum, "Design of exponential observers for nonlinear systems by embedding," International Journal of Robust and Nonlinear Control, vol. 14, pp. 273-288, 2004. 